\title{
Artificial Neural Network Model for Tool Condition Monitoring in Stone Drilling
}

\author{
Danko Brezak ${ }^{1, a}$, Tomislav Staroveski ${ }^{2, b}$, Ivan Stiperski ${ }^{3, c}$, Miho Klaic $^{4, d}$ \\ and Dubravko Majetic ${ }^{5, e}$ \\ ${ }_{1,2,3,4,5}$ Faculty of Mechanical Engineering and Naval Architecture, University of Zagreb, Ivana \\ Lucica 5, Zagreb, HR-.10000, Croatia \\ adanko.brezak@fsb.hr, btomislav.staroveski@fsb.hr, 'stiperski.ivan@gmail.com, \\ dmiho.klaic@fsb.hr, ${ }^{\mathrm{e}}$ dubravko.majetic@fsb.hr
}

Keywords: Stone drilling, tool wear classification, neural networks

\begin{abstract}
This paper explores the possibility of tool wear classification in stone drilling. Wear model is based on Radial Basis Function Neural Network which links tool wear features extracted from motor drive current signals and acoustic emission signals with two wear levels - sharp and worn drill. Signals were measured during stone drilling under different cutting conditions, and then filtered before tool wear features extraction. Features were obtained from time and frequency domain. They have been analyzed individually and in combinations. The results indicate tool wear monitoring capacity of the proposed model in stone drilling, and its potential for simple and costeffective integration with $\mathrm{CNC}$ machine tools.
\end{abstract}

\section{Introduction}

Tool wear monitoring is one of the most important segments in the development of fully automated and highly autonomous CNC machine tools. Except in machine tool diagnostics, it is also necessary in the implementation of machining process control systems which could prevent tool breakage and/or maintain predefined tool wear dynamic $[1,2]$. Tool wear monitoring in drilling has been continuously in the research focus for the past 20 years. A number of machine learning algorithms, sensor combinations and tool wear features have been analyzed and proposed, mainly using metal and composite materials [3]. Only a few studies considered wear monitoring in stone machining. They have usually included wear identification of diamond tools applied in cutting and/or milling using cutting forces sensors [4-7].

The aim of this study was to analyze capabilities of neural network-based tool wear classification model in stone drilling using cost-effective combination of internal drive signals or currents (instead of cutting forces) and acoustic emission sensor. For this purpose, a type of Radial Basis Function Neural Network (RBF NN) algorithm for solving classification types of problems has been chosen. This type of neural network is known for its learning in one step and a capability of simple and quick hidden layer structure adaptation. Experiments were conducted using a custom-made machine tool testbed with open architecture control platform.

\section{Experimental Work}

Machine Tool Testbed. Experimental work has been performed using the three-axis bench-top CNC mini milling machine with an internal and external measurement systems (Fig. 1). The machine has been retrofitted with the $0.4 \mathrm{~kW}(1.27 \mathrm{Nm})$ permanent magnet synchronous motors with integrated incremental encoders (Mecapion SB04A), corresponding motor controllers (DPCANIE-030A400 and DPCANIE-060A400), ball screw assemblies, and LinuxCNC open architecture control (OAC) system [8]. Considering the nature of the drilling process, two types of signals were sampled from those controllers: vertical or $\mathrm{Z}$-axis feed drive current $\left(\mathrm{I}_{\mathrm{Z}}\right)$ and main spindle current $\left(\mathrm{I}_{\mathrm{MS}}\right)$. Beside motor drive currents, acoustic emission signals (AE) were also measured using 8152B piezoelectric AE sensor and 5125 coupler (Kistler) connected to PCI- 
DAS4020/12 data acquisition board. Customized measurement software developed in the LabView environment was used for cutting conditions setup, storage of the acquired signals and NC drilling cycle program generation. Each measurement started by issuing the trigger signal from the CNC control system to the measurement systems. Direct observations of the drill cutting edges were made using the industrial camera type DMK41AF02 equipped with the telecentric lenses type TC2309. Twist drill type BOSCH CYL-9 (5 mm in diameter) was used to drill $10 \mathrm{~mm}$ deep holes in Adria Grigio Machiato stone samples.
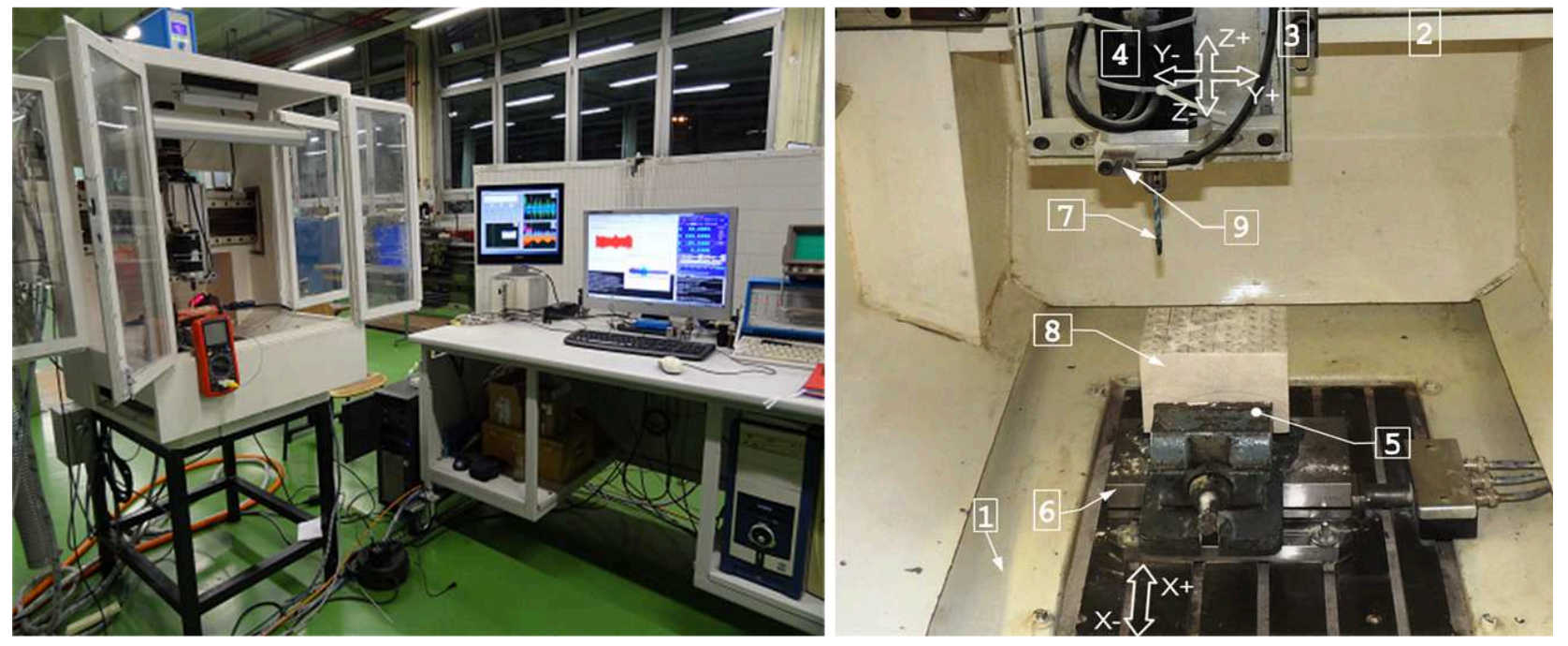

Fig. 1. Experimental setup - work area and sensor placement. (1) X axis feed drive; (2) Y axis feed drive; (3) $\mathrm{Z}$ axis feed drive; (4) main spindle drive; (5) workpiece fixture; (6) three axis force sensor; (7) test drill; (8) stone workpiece; (9) AE sensor

Data Acquisition and Signal Processing. Motor drive current signals were sampled continuously at $1 \mathrm{kHz}$, and $\mathrm{AE}$ signals at $2 \mathrm{MHz}(0.1 \mathrm{~s}$ sample duration per hole). Signals were measured with sharp (SD) and worn drill (WD) using 9 combinations of cutting speeds $(10 ; 30 ; 50 \mathrm{~m} / \mathrm{min})$, and feed rates $(0.05 ; 0.1 ; 0.15 \mathrm{~mm} / \mathrm{rev})$, which were chosen according to the tool manufacturer recommendations for this type of drill. Those cutting speeds correspond to spindle speeds of 636.6 rpm, $1909.8 \mathrm{rpm}$ and $3183.09 \mathrm{rpm}$, respectively. For each combination of machining parameters experiment was randomly repeated 10 times. Measurements are first taken while drilling with completely sharp drill and then repeated using the worn drill. After completing the measurements with the sharp drill, it was then used to drill a number of cycles until it completely worn out. Flank wear and cracks were observed as a dominant wear features (Fig. 2).

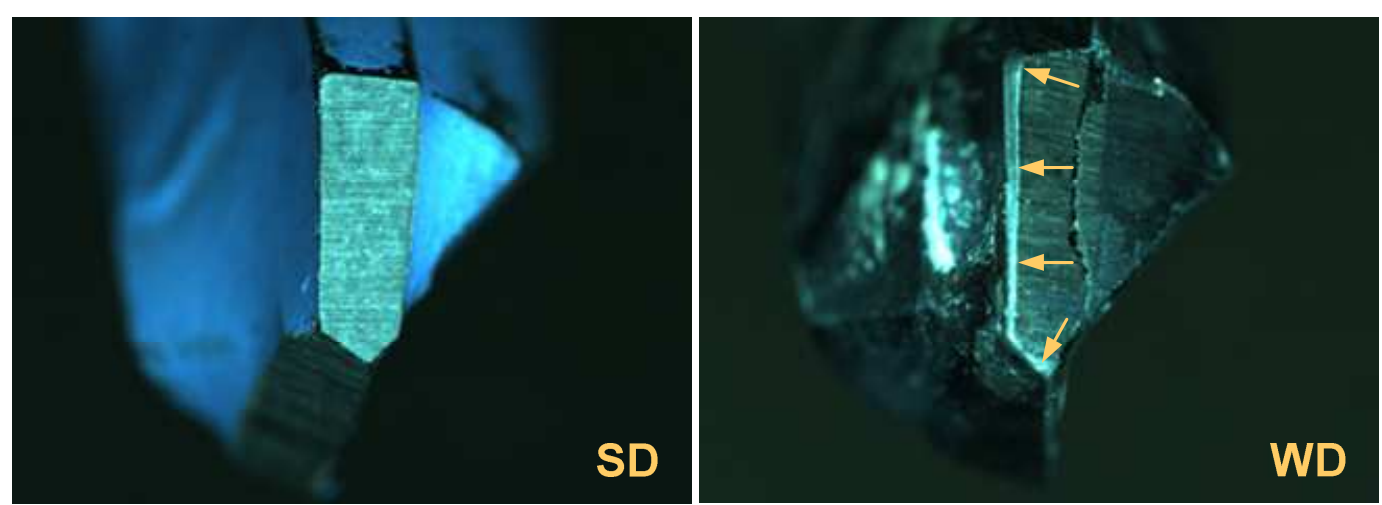

Fig. 2. Images of cutting edges after drilling with sharp (SD) and worn drill (WD) with observable (arrows pointing to) flank wear area 
Before extracting tool wear features, both types of signals were filtered. In the case of current signals, Butterworth low-pass filter with $2 \mathrm{~Hz}$ cut-off frequency was applied. This frequency was chosen after spectral analyzes of signals using Fast Fourier Transform (FFT). AE signals were filtered with the Butterworth band-pass filter (frequency range $40-500 \mathrm{kHz}$ ), which was in accordance with the specified frequency or measurement range of the utilized sensor.

Tool Wear Features. After filtration, 8 features were extracted from current signals. First two features were maximum values of both types of signals (Max_IZ, Max_ $\mathrm{I}_{\mathrm{MS}}$ ). They were calculated based on an average value of the $10 \%$ of the highest current values, thus neutralizing eventual occurrence of transient spikes. The next two features (Area_ $\mathrm{I}_{Z}$, Area_ $\mathrm{I}_{\mathrm{MS}}$ ) were areas under current curves related to the machining time. This type of features is closely related to the total amount of electric energy used in the cutting process. The remaining four features were from the frequency domain: power of spectral components related to the rotation frequency - $R F$ (P_RF_I,$P_{-}$RF_IMS) and cutting edges frequency - $\mathrm{CF}\left(\mathrm{P}_{-} \mathrm{CEF} \mathrm{I}_{Z}, \mathrm{P}_{-} \mathrm{CEF} \mathrm{I}_{\mathrm{MS}}\right)$ [9]. Since drill has two cutting edges, $\mathrm{CEF}$ was twice as high as RF. Those features were obtained using the FFT algorithm.

Features from AE signals were all extracted from the frequency domain. Measured frequency range $(40-500 \mathrm{kHz})$ was divided into 7 frequency ranges $(50-100 \mathrm{kHz} ; 100-150 \mathrm{kHz} ; \ldots ; 350-400$ $\mathrm{kHz}$ ), and energy of every range has been taken as a drill wear feature [10]

$$
\psi^{2}=\int_{\mathrm{fl}}^{\mathrm{fh}} \mathrm{S}_{\mathrm{y}} \mathrm{df},
$$

where $S_{y}$ is the one-sided PSD function of the AE signal, while $f_{1}$ and $f_{h}$ are lower and upper frequency values chosen to reflect the energy in the range of interest. Altogether, 15 features have been extracted from both types of signals (Table 1).

Table 1. List of Drill Wear Features

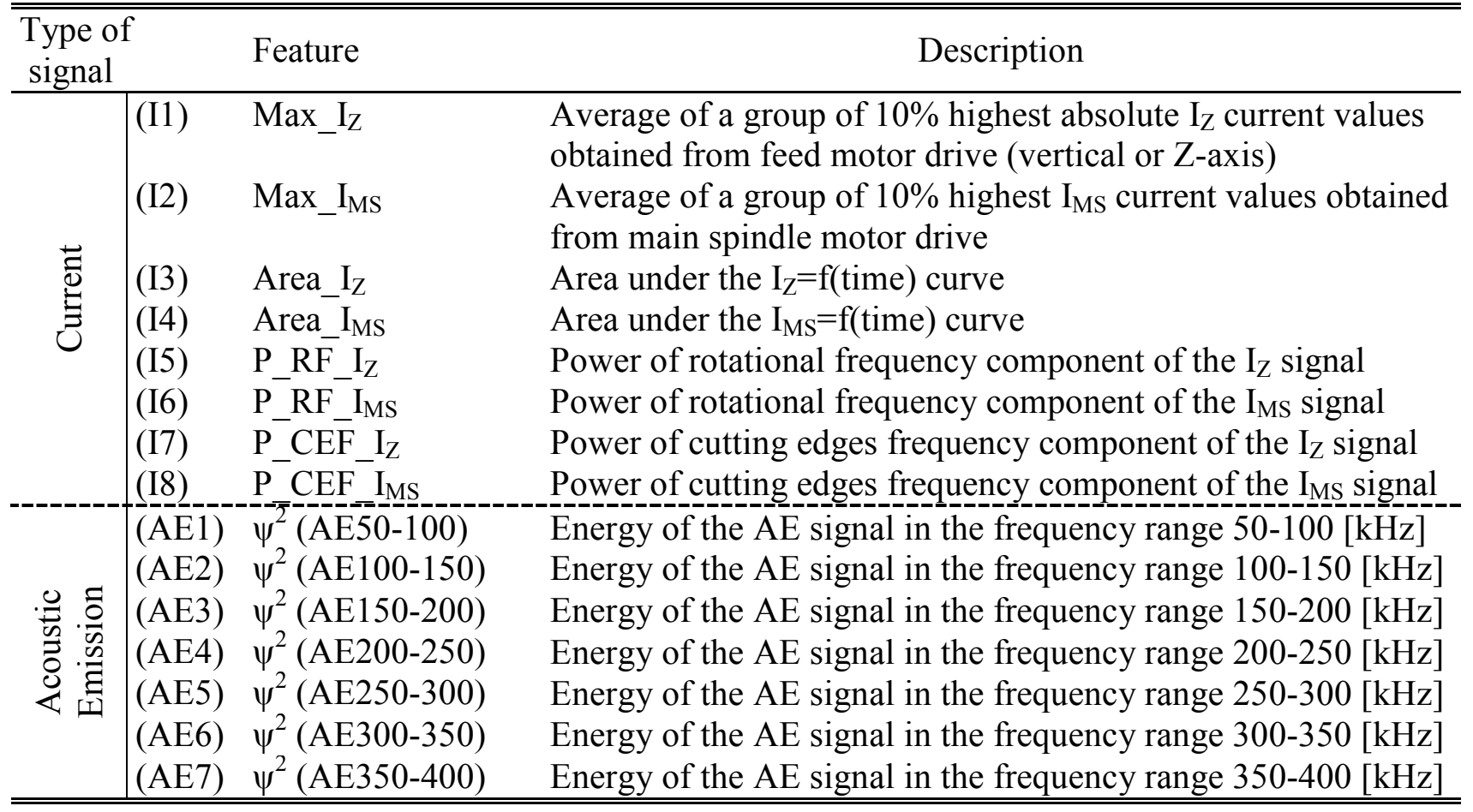




\section{RBF Neural Network}

Chosen NN algorithm is based upon a well-known feedforward three-layered RBF NN architecture, where the matrix/vector of synaptic weights $\mathbf{c}$ is calculated in the learning phase using the expression

$$
\mathbf{c}=\mathbf{H}^{+} \mathbf{y}
$$

where $\mathbf{y}$ stands for the matrix/vector of desired output values and $\mathbf{H}^{+}$is Moore - Penrose pseudoinverse of the matrix of hidden layer neuron RBF outputs or activation function outputs $(\mathbf{H})$. The pseudoinverse is defined as follows

$$
\mathbf{H}^{+}=\left(\mathbf{H}^{\mathrm{T}} \mathbf{H}\right)^{-1} \mathbf{H}^{\mathrm{T}} .
$$

In the testing phase, the matrix of desired output values $\mathbf{y}$ is obtained from the expression

$$
\mathbf{y}=\mathbf{H c} \text {. }
$$

Elements of matrix $\mathbf{H}$ are determined according to the expression

$$
H_{i j}=e^{-\frac{1}{2} r_{i j}^{2}}, i=1, \ldots, N, j=1, \ldots, K
$$

where $r_{i j}$ is the Mahalanobis distance between vector composed from $i$ th element of all input vectors (tool wear features) and $j$ th hidden layer neuron (or hidden layer neuron center). Squared Mahalanobis distance is calculated using the expression

$$
\mathrm{r}_{\mathrm{ij}}^{2}=\left(\mathbf{x}_{\mathrm{i}}-\mathbf{t}_{\mathrm{j}}\right)^{\mathrm{T}} \boldsymbol{\Sigma}_{\mathrm{j}}^{-1}\left(\mathbf{x}_{\mathrm{i}}-\mathbf{t}_{\mathrm{j}}\right),
$$

where $\Sigma_{\mathrm{j}}$ is a covariance matrix belonging to the group of learning samples that are connected to the $j$ th hidden layer neuron, $\mathbf{x}_{\mathrm{i}}$ is the L-dimensional vector composed from $i$ th element of all $\mathrm{L}$ input vectors and $\mathbf{t}_{\mathbf{j}}$ is L-dimensional vector of the $j$ th hidden layer neuron center. Covariance matrix is quadratic matrix with non-zero elements (squared $\sigma$ vector components) on main diagonal and zeros elsewhere,

$$
\boldsymbol{\Sigma}_{\mathrm{j}}=\left[\begin{array}{ccc}
\sigma_{1}^{2} & 0 & 0 \\
0 & \ddots & 0 \\
0 & 0 & \sigma_{\mathrm{L}}^{2}
\end{array}\right]
$$

Since every center is defined in the learning phase based on the group of network input elements, vector $\sigma$ is composed from the maximal Euclidian distances between learning samples belonging to the analyzed group and the center of that group, regarding to all (L) dimensions separately,

$$
\left.\sigma_{\mathrm{g}}\right|_{\mathrm{j}}=\left.\max \left\{\left\|\mathrm{z}_{\mathrm{pg}}-\mathrm{t}_{\mathrm{g}}\right\|, \mathrm{p}=1, \ldots, \mathrm{LK}_{\mathrm{G}}\right\}\right|_{\mathrm{j}}, \mathrm{g}=1, \ldots, \mathrm{L}
$$

where $z_{p g}$ is the gth component of the $p$ th sample of the $j$ th group which is defined with $\mathrm{LK}_{\mathrm{G}}$ numbers of samples, and $t_{\mathrm{g}}$ is $g$ th component of the $j$ th group center vector $(j$ th hidden layer neuron center vector).

Hidden layer neuron centers are defined using a method which helps teacher to quickly determine network structure regarding to the nature of the learning problem and desirable network 
generalization characteristics. Grouping of tool wear features or network input elements and centers calculations are based on the parameter $\beta_{C}$. Higher $\beta_{C}$ reduces the number of hidden layer neurons and vice versa (for $\beta_{C}=0$ every input vector forms one hidden layer neuron or its center). Hidden layer configuration method is in detail explained in [11].

\section{Results}

With 9 combinations of machining parameters, 10 measurements for each combination, and 2 drill wear levels or classification groups, 180 sets of samples were collected in total. Five out of 10 samples of repetitive measurements for each combination of machining parameters were used in the learning phase, and the remaining five participated in the formation of data sets used in the testing phase of the RBF NN classifier.

In order to analyze capacity of chosen features for drill wear classification, and to find combination(s) which provide the best classification performance, learning/testing procedure was divided into several steps. In the first step, every feature has been analyzed separately using full hidden layer structure $\left(\beta_{C}=0\right)$. Based on these first results, further analyzes of different feature combinations have been performed (also with $\beta_{C}=0$ ). Feature combinations have expectedly achieved higher classification accuracy than the individual features, and the results of chosen combinations are presented in Table 2. All presented results were achieved using cutting speed and feed rate as two additional NN inputs, and classification success rate in the learning phase was $100 \%$.

Table 2. Classification Results - Accurately Classified Samples, (\%)

\begin{tabular}{|c|c|c|c|c|c|c|c|}
\hline \multirow{2}{*}{ Feature } & \multicolumn{6}{|c|}{ TEST } & \multirow{2}{*}{$\begin{array}{l}\text { RBF NN } \\
\text { structure }\end{array}$} \\
\hline & T1 & $\mathrm{T} 2$ & T3 & T4 & T5 & Avg. & \\
\hline $\mathrm{I} 1+\mathrm{I} 2+\mathrm{I} 3+\mathrm{I} 4$ & 88.9 & 94.4 & 88.9 & 94.4 & 100 & 93.3 & $6-90-2$ \\
\hline $\mathrm{I} 5+\mathrm{I} 6+\mathrm{I} 7+\mathrm{I} 8$ & 66.7 & 83.3 & 83.3 & 66.7 & 83.3 & 76.7 & $6-90-2$ \\
\hline $\mathrm{I} 1+\mathrm{I} 2+\ldots+\mathrm{I} 8=\Sigma \mathrm{I}$ & 83.3 & 88.9 & 94.4 & 72.2 & 94.4 & 86.6 & $10-90-2$ \\
\hline AE4+AE5 & 88.9 & 77.8 & 83.3 & 72.2 & 88.9 & 82.2 & $4-90-2$ \\
\hline $\mathrm{AE} 1+\mathrm{AE} 2+\ldots+\mathrm{AE} 7=\Sigma \mathrm{AE}$ & 77.8 & 77.8 & 77.8 & 77.8 & 61.1 & 74.5 & $9-90-2$ \\
\hline $\mathrm{I} 1+\mathrm{I} 2+\mathrm{I} 3+\mathrm{I} 4+\mathrm{AE} 4+\mathrm{AE} 5$ & 83.3 & 94.4 & 94.4 & 100 & 94.4 & 93.3 & $8-90-2$ \\
\hline$\Sigma \mathrm{I}+\Sigma \mathrm{AE}$ & 83.3 & 88.9 & 94.4 & 72.2 & 83.3 & 84.4 & $17-90-2$ \\
\hline $\mathrm{I} 1+\mathrm{I} 2+\mathrm{I} 3+\mathrm{I} 4+\mathrm{AE} 4+\mathrm{AE} 5$ & 88.9 & 94.4 & 88.9 & 94.4 & 88.9 & 91.1 & $8-58-2\left(\beta_{C} \neq 0\right)$ \\
\hline
\end{tabular}

Combination of time domain features extracted from $I_{Z}$ and $I_{M S}$ current signals has shown the highest average classification accuracy based on 5 analyzed tests (93.3\%). The same result was achieved when this combination was further extended by two extra features from AE signals (AE4, AE5). These two features accomplished the best individual classification accuracy among the features from AE signals. Other combinations did not manage to reduce average classification error below $10 \%$, but their results are still more than acceptable.

At the end, the best combination of features extracted from both types of signals was analyzed once more with the reduced number of hidden layer neurons $\left(\beta_{C}>0\right)$ to find out the RBF NN structure still capable to provide satisfactory generalization characteristics. It can be noticed that RBF NN with the number of hidden layer neurons decreased by $30 \%$ (58 vs. 90) managed to maintain classification accuracy higher than $90 \%$.

\section{Summary}

In this work a type of Radial Basis Function Neural Network algorithm has been applied for tool wear classification under different cutting conditions in stone drilling. Tool wear features were obtained from Z-axis (vertical axis) feed drive current, main spindle drive current, and acoustic 
emission signals. They were then used for classification of drill wear into two groups: sharp and completely worn drill. Every feature was analyzed separately and in combinations.

The accomplished results suggest potential of the analyzed model for tool wear monitoring during stone drilling. Most successful model outputs were achieved using only features from servomotor current signals, thus supporting the idea of reliable and cost-effective monitoring system without the usage of force sensor. Practically identical result has been achieved using the combination of selected features from current and acoustic emission signals. This is also very important, since it is a wide known fact that tool wear is highly non-linear and partially stochastic process which cannot be reliably identified and monitored in the real industrial environment using only one type of signal. Furthermore, AE sensors are low-cost and easily integrated into the machine tool structure.

The proposed tool wear model is very simple to implement in the CNC control system and shows the potential for tool wear monitoring in drilling. It was analyzed with experimental data obtained while drilling a single type of stone material with a single type of twist drill. Future experimental work will therefore be extended to different types of stone materials, drill diameters and geometries, as well as more than two drill wear levels.

\section{References}

[1] S. Liang, R.L. Hecker, and R.G. Landers: ASME Journal of Manufacturing Science and Engineering, Vol. 126 (2004), pp. 297-310.

[2] R. Teti, K. Jemielniak, G. O'Donnell, and D. Dornfeld: CIRP Annals - Manufacturing Technology, Vol. 59 (2010), pp. 717-739.

[3] E. Jantunen: Journal of Machine Tools and Manufacture, Vol. 42 (2002), pp. 997-1010.

[4] W. Polini and S. Turchetta: The International Journal of Advanced Manufacturing Technology, Vol. 35 (2007), pp 454-467.

[5] W. Polini and S. Turchetta: Advances in Mechanical Engineering, Vol. 2009 (2009).

[6] S. Turchetta: The International Journal of Advanced Manufacturing Technology, Vol. 61 (2012), pp. 441-448.

[7] J. Kenda and J. Kopac: Strojniški vestnik - Journal of Mechanical Engineering, Vol. 55 (2009), pp. 775-780.

[8] T. Staroveski, D. Brezak, T. Udiljak, and D. Majetic: Annals of DAAAM International 2011, Vol. 22 (2011), pp. 0023-0024.

[9] P.W. Prickett, C. Johns: International Journal of Machine Tools \& Manufacture, Vol. 1 (1999), pp.105-122.

[10]C. Scheffer, P.S. Heyns, and F. Klocke: International Journal of Machine Tools \& Manufacture, Vol. 43 (2003), pp. 973-985.

[11]D. Brezak, T. Udiljak, K. Mihoci, D. Majetic, B. Novakovic, and J. Kasac: Proceedings of International Joint Conference on Neural Networks - IJCNN (2004), pp. 1859-1863. 